\title{
Missing the forest for the trees: can we use quality of care indicators to determine when chemotherapy administration is appropriate for vulnerable populations with cancer?
}

\author{
Christine Sam ${ }^{1}$, Tawee Tanvetyanon ${ }^{2}$ \\ ${ }^{1}$ Department of Individualized Cancer Management, Senior Adult Oncology Program, ${ }^{2}$ Department of Thoracic Oncology, H. Lee Moffitt Cancer \\ Center, Tampa, FL, USA \\ Correspondence to: Dr. Christine Sam, MD. Senior Adult Oncology Program, Moffitt Cancer Center, 12902 Magnolia Drive, Tampa, FL 33612, USA. \\ Email: christine.sam@moffitt.org. \\ Comment on: Ellis RJ, Schlick CJR, Feinglass J, et al. Failure to administer recommended chemotherapy: acceptable variation or cancer care quality \\ blind spot? BMJ Qual Saf 2020;29:103-12.
}

Received: 31 March 2020; Accepted: 06 May 2020; Published: 25 September 2020.

doi: 10.21037/jhmhp-2020-49

View this article at: http://dx.doi.org/10.21037/jhmhp-2020-49

Adjuvant chemotherapy when used in the right setting at the right time can be life-saving. One of the quality-of-care measures set forth by the American College of Surgeons Commission on Cancer $(\mathrm{CoC})$ is geared toward the timely administration of adjuvant chemotherapy after surgery. For example, in the setting of non-small cell lung cancer (NSCLC), chemotherapy should be administered within 4 months preoperatively, or recommended for all surgically resected cases with pathologic, lymph node-positive NSCLC. In a recent large study based on data obtained from the National Cancer Database, the researchers found that $3.5 \%$ to $10.7 \%$ of patients were recommended to have chemotherapy but did not actually receive it. While the $\mathrm{CoC}$ measure will consider this as acceptable as long as chemotherapy has been recommended, the gap may reflect a failure of healthcare system to help patients overcome barriers to chemotherapy. For example, patients may face challenges such as lack of transportation, out of pocket expense, or poor communication, thus resulting in the missed opportunity to receive the prescribed chemotherapy. Nevertheless, we feel that it will be difficult to quantify the magnitude of failure via retrospective medical record review. Specific reasons why oncologists decide not to prescribe chemotherapy may be nuanced or not documented in the medical records, making it looks as though chemotherapy has been recommended. Moreover, even when chemotherapy is intended, patients may decide later not to have treatment. It should be noted that, even in an ideal environment such as prospective clinical trial, patients who are assigned to receive treatment but not actually treated will also need to be included under the treatment arm per intention-to-treat analysis. While the rate of failure to receive prescribed chemotherapy may not be very useful for comparison across hospitals, it can be used as a rough baseline benchmark for each hospital. In summary, while there may really be a quality of care blind spot, one should avoid using the quality measure related to chemotherapy utilization blindly to pressure hospitals to administer more chemotherapy. Due to the potential toxicity from chemotherapy, doing so can be detrimental, leading to poorer outcomes in the end for every party.

Studies have shown that timely delivery of adjuvant chemotherapy in appropriate patients can lead to an improvement in survival and decreased recurrence rates in various cancers. For example, post-operative treatment with cisplatin-based doublet chemotherapy for selected patients with NSCLC can increase the absolute cure rate by about $5 \%$ (1). In a large randomized clinical trial published in 2004 enrolling over 1,800 patients, the intention-totreat analysis showed that about $40 \%$ of those who were randomized to chemotherapy remained free of cancer at 5 years, compared with only $35 \%$ among those who randomized to observation alone. Currently many treatment guidelines such as the National Comprehensive Cancer Network guideline are available to help remind clinicians about when and how to prescribe appropriate chemotherapy 
in various cancers. Above this, quality measures have also been developed to ensure that these standards of care are being followed at the hospital level. It should be noted that, in general, good quality measures should be evidence-based, objectively measurable, and actionable by the hospital or the responsible party (2). After all, if nothing can be done about a problem, identifying or tracking the problem will not help solve it.

Appropriate administration of adjuvant chemotherapy is endorsed by the National Quality Forum, and metrics have been recognized by accrediting organizations including the Centers for Medicare and Medicaid Services as well as the American College of Surgeons CoC to measure quality of care among hospitals. For example, in the setting of NSCLC, the CoC states that systemic chemotherapy should be administered within 4 months to day preoperatively or day of surgery to 6 months postoperatively, or it is recommended for surgically resected cases with pathologic, lymph node-positive NSCLC (3). In the current definition, either recommendation to have chemotherapy or the actual receipt of chemotherapy is acceptable as quality care. While this metric seems fair in face value, a concern has been raised by a recently published study by Ellis and colleagues it may miss patients who "fell through the cracks," i.e., did not pursue treatment due to socioeconomic factors such as poor medical literacy, language barriers, and financial impediments (4). Given that hospitals should have resources to assist patients to overcome these barriers, allowing just recommendation to receive chemotherapy but not necessarily the actual receipt of chemotherapy to be counted toward quality of care metric could potentially lead to an overly optimistic estimate of care quality.

In their study, chemotherapy utilization among hospitals in the National Cancer Database was recalculated based on an alternate definition which did not allow just a recommendation alone. The cancers assessed included early-stage hormone negative breast, colon, and lung cancer. The gaps between the two calculations were actually small. Overall, $3.5 \%$ of patients with breast, $6.6 \%$ with colon and $10.7 \%$ with lung cancers failed to receive chemotherapy offered by their care providers. On multivariable analysis, advanced age and uninsured/ Medicaid status were significantly associated with failure to receive adjuvant chemotherapy. For example, the overall rate of failure in colon cancer was $10.5 \%$ among patients $>70$ years old compared to just $3.4 \%$ among those $<55$ years old (adjusted odds ratio 3.56, 95\% confidence interval: 3.23 to 3.92 ). Furthermore, the investigators found that there was a wide variation among hospitals, some as high as $20-40 \%$ failure to receive recommended chemotherapy. Other characteristics that were significantly associated with higher failure rates included non-Hispanic black race (breast and colon cancers), lower income (breast and colon cancers), and higher comorbidity index score (colon cancer).

Indeed, Ellis et al. does raise a valid concern about the possibility that certain vulnerable populations may have a higher rate of chemotherapy failure-to-delivery due to socioeconomic factors. There is extensive literature regarding the inferior cancer care outcomes in vulnerable populations such as the elderly, racial minorities, and patients of lower socioeconomic status $(5,6)$. While improving cancer care in vulnerable populations needs to be a priority for health systems in order to deliver equitable care, considerations will need to be given with regard to the validity of measurement, availability of solution, and relative impact of the solution, especially in the face of other competing priorities for healthcare improvement.

With regard to validity of the measurement, the institution of quality metrics can only capture aggregate data retrospectively, and therefore, will potentially miss individualized treatment decisions between a patient and oncologist. Appropriate cancer care for each patient can be nuanced, taking into account multiple factors such as age, comorbidities, psychosocial circumstances, and personal preferences. Regardless of the reasons, sometimes this may not be documented in the medical records, making it appears as though chemotherapy was offered but not undertaken. When the setting is not right for chemotherapy, one of the best things for a treating physician to do is to not give chemotherapy. Because chemotherapy can bring about serious toxicities, its utilization may not be an ideal quality of care indicator to be used to judge the appropriateness of care. Giving chemotherapy when the setting will appear right to the retrospective reviewer's eyes but not right for the treating physician and patient at the time of decision making can actually make the outcome worse in the end. Clearly this quality of care metric is not as simple as wrongsite surgery or infant vaccination.

Even when there is a clear intention to treat by physicians, chemotherapy may be refused or not feasible because of unforeseen circumstances such as rapid disease progression. It should be noted that, in the clinical trial of adjuvant chemotherapy for NSCLC1, about $8 \%$ of patients who were randomized to receive chemotherapy did not receive any chemotherapy at all for various reasons (1). Nevertheless, the analysis was conducted according to 
intention to treat principle which is widely accepted as good practice in statistical analysis. Additionally, when interpreting clinical trial, generalizability across patient populations must be kept in mind. Elderly patients are underrepresented in clinical trials, and patients that are included on the trials usually have excellent performance status, which may not reflect the patient seen day to day in the real world. Those with multiple comorbidities, functional or cognitive impairments, common issues in elderly patients, are either excluded or not actively enrolled onto clinical trials. As a result, applying "standard" guidelines to blindly can be dangerous and lead to greater toxicities and quality of life detriment than reported in the clinical trials. Elderly patients are also more likely to prize quality of life as part of their health goals, and many decline chemotherapies that may carry immediate high risk of toxicity in trade of future longevity.

Although the quality of care metric based on adjuvant chemotherapy is susceptible to variation in socioeconomic or cultural status across regions, the metric can still be useful as a baseline benchmark for each hospital. For instance, among oncology practices in the Florida Initiative for Quality Cancer Care project, which serves a large number of elderly patients, the quality of care in NSCLC was assessed using a set of selected quality indicators including adjuvant chemotherapy (7). The general adherence to general quality indicators such as documentation of TNM stage was about $95 \%$, while the use of adjuvant chemotherapy was much lower and varied widely by practices. However, following implementation of quality improvement program each practice can have an opportunity to improve on its own baseline performance serving as benchmark (8).

In summary, we feel that the inclusion of patients who were recommended but did not ultimately receive chemotherapy may not necessarily represent a blind spot in the current quality metric definition. This metric while important and useful, has a clear limitation in itself. While some patients may really fall through the cracks, it is possible that many do have undocumented reasons not to undergo chemotherapy. Furthermore, while vulnerable populations such as elderly, minority and socioeconomically disadvantaged populations may have lower rates of timely chemotherapy administration, this does not necessarily reflect a failure of healthcare providers or hospitals. In fact, attempting to increase chemotherapy utilization among certain populations may lead to adverse consequences. Focusing solely on chemotherapy delivery rather than overall health outcomes is akin to missing the forest for the trees. Nonetheless, efforts should be made to ensure that all patients have adequate education about adjuvant treatment as a treatment option and mitigate any known barriers to treatment related to treatment. For this purpose, patient navigation programs integrating multidisciplinary teams such as social workers and case managers can be helpful. Finally, to address the problem of patients falling through the cracks, specific quality of care metric related to social infrastructure can be used.

\section{Acknowledgments}

Funding: None.

\section{Footnote}

Provenance and Peer Review: This article was commissioned by the editorial office, Fournal of Hospital Management and Health Policy. The article did not undergo external peer review.

Conflicts of Interest: Both authors have completed the ICMJE uniform disclosure form (available at http://dx.doi. org/10.21037/jhmhp-2020-49). The authors have no conflicts of interest to declare.

Ethical Statement: The authors are accountable for all aspects of the work in ensuring that questions related to the accuracy or integrity of any part of the work are appropriately investigated and resolved.

Open Access Statement: This is an Open Access article distributed in accordance with the Creative Commons Attribution-NonCommercial-NoDerivs 4.0 International License (CC BY-NC-ND 4.0), which permits the noncommercial replication and distribution of the article with the strict proviso that no changes or edits are made and the original work is properly cited (including links to both the formal publication through the relevant DOI and the license). See: https://creativecommons.org/licenses/by-nc-nd/4.0/.

\section{References}

1. Arriagada R, Bergman B, Dunant A, et al. Cisplatinbased adjuvant chemotherapy in patients with completely resected non-small-cell lung cancer. N Engl J Med 2004;350:351-60. 
2. Rubin HR, Pronovost P, Diette GB. From a process of care to a measure: the development and testing of a quality indicator. Int J Qual Health Care 2001;13:489-96.

3. American College of Surgeon. CoC Quality of Care Measures. Available online at Available online: https://www.facs.org/quality-programs/cancer/ncdb/ qualitymeasurescocweb. Access verified March 28, 2020.

4. Ellis RJ, Schlick CJR, Feinglass J, et al. Failure to administer recommended chemotherapy: acceptable variation or cancer care quality blind spot? BMJ Qual Saf 2020;29:103-12.

5. Green AK, Aviki EM, Matsoukas K, et al. Racial disparities in chemotherapy administration for early-stage breast

doi: 10.21037/jhmhp-2020-49

Cite this article as: Sam C, Tanvetyanon T. Missing the forest for the trees: can we use quality of care indicators to determine when chemotherapy administration is appropriate for vulnerable populations with cancer? J Hosp Manag Health Policy 2020;4:19. cancer: a systematic review and meta-analysis. Breast Cancer Res Treat 2018;172:247-63.

6. Duma N, Idossa DW, Durani U, et al. Influence of Sociodemographic Factors on Treatment Decisions in Non-Small-Cell Lung Cancer. Clin Lung Cancer 2020;21:e115-e129.

7. Tanvetyanon T, Corman M, Lee JH, et al. Quality of care in non-small-cell lung cancer: findings from 11 oncology practices in Florida. J Oncol Pract 2011;7:e25-e31.

8. Tanvetyanon T, Lee JH, Fulp WJ, et al. Changes in the care of non-small-cell lung cancer after audit and feedback: the Florida initiative for quality cancer care. J Oncol Pract 2014;10:e247-e254. 